MORETTI, C.L.; SARGENT, S.A.; BALABAN, M.O.; PUSCHMANN, R. Nariz eletrônico: tecnologia não destrutiva para a detecção de desordem fisiológica causada por impacto em frutos de tomate. Horticultura Brasileira, Brasília, v. 18, n. 1, p. 20-23, março 2.000.

\title{
Nariz eletrônico: tecnologia não-destrutiva para a detecção de desordem fisiológica causada por impacto em frutos de tomate.
}

\author{
Celso Luiz Moretti ${ }^{1}$; Steven Alonzo Sargent ${ }^{1}$; Murat O. Balaban ${ }^{2}$, Rolf Puschmann ${ }^{3}$. \\ ${ }^{1}$ Embrapa Hortaliças, C. Postal 218, 70.359-970; Brasília-DF; ${ }^{2}$ Universidade da Flórida, Gainesville, 32611, USA; ${ }^{3}$ UFV, Depto. de \\ Biologia Vegetal, 36.571-000,Viçosa-MG.
}

\section{RESUMO}

Frutos de tomate (Lycopersicon esculentum Mill.), cv. Solar Set, foram colhidos no estádio verde-maduro ( $100 \%$ da superfície com coloração verde) e tratados com $100 \mu \mathrm{L} . \mathrm{L}^{-1}$ de etileno gasoso a $20^{\circ} \mathrm{C}$ Após atingirem o estádio verde-rosado (menos de $10 \%$ da superfície do fruto com coloração vermelha ou amarelo-tanino), parte dos frutos foram submetidos a uma queda de $0,40 \mathrm{~m}$ sobre uma superfície plana e lisa. Posteriormente, os frutos submetidos ao impacto e os não-submetidos foram armazenados a $20^{\circ} \mathrm{C}$ e $85-95 \%$ de umidade relativa até estarem completamente amadurecidos. Os frutos com e sem injúrias mecânicas foram então colocados individualmente no frasco de amostragem do "nariz eletrônico" e os doze sensores iniciaram a detecção dos compostos emanados pelos frutos. Os dados foram submetidos à análise discriminante multivariada. $\mathrm{O}$ grau de dissimilaridade entre os tratamentos foi definido utilizando-se a distância de Mahalanobis. As diferenças encontradas nos frutos com e sem injúria mecânica foram significativas $(\mathrm{P}<0,0041)$. A distância de Mahalanobis entre grupos (28,19 unidades) foi um indicativo dramático das diferenças encontradas entre os dois grupos de frutos. A análise do desempenho do "nariz eletrônico" demonstrou que o equipamento é uma ferramenta útil para classificar, não-destrutivamente, tomates expostos a condições extremas de manuseio pós-colheita, como injúrias mecânicas.

Palavras-chave: Lycopersicon esculentum, armazenamento, injúria mecânica, pós-colheita.

\begin{abstract}
Electronic nose: a non-destructive technology to screen tomato fruit with internal bruising.

Tomato (Lycopersicon esculentum Mill) fruits, 'Solar Set', were harvested at the mature-green stage (green color in 100\% of the fruit surface) and gassed with $100 \mathrm{~mL}^{-1}$ of ethylene at $20^{\circ} \mathrm{C}$. At the breaker stage (less than $10 \%$ of the fruit surface is red or tanninyellowish), fruit were dropped from a $40 \mathrm{~cm}$ height onto a smooth surface. Following impact, fruits were stored at $20^{\circ} \mathrm{C}$ and $85-95 \%$ relative humidity until table-ripe stage. Bruised and unbruised fruit were then placed individually inside the electronic nose-sampling vessel and the twelve conducting polymer sensors were lowered into the vessel and exposed to the volatile given off by the fruit. Data were analyzed employing multivariate discriminant analysis (MVDA), which maximizes the variance between treatments. The degree of dissimilarity was defined using the Mahalanobis distance The differences found between bruised and unbruised fruit were highly significant $(\mathrm{P}<0.0041)$. The Mahalanobis distance between groupings (28.19 units) was a dramatic indicative of the differences between the two treatments. The electronic nose proved to be a useful tool to nondestructively identify and classify tomato fruit exposed to harmful post-harvest practices such as mechanical injuries.
\end{abstract}

Keywords: Lycopersicon esculentum, mechanical injury, postharvest, storage.

(Aceito para publicação em 31 de janeiro de 2.000)

A ocorrência de injúrias mecânicas de impacto, vibração e compressão está geralmente associada a todas as etapas do manuseio pós-colheita dos diversos produtos hortícolas. A severidade e os danos decorrentes das injúrias mecânicas variam de acordo com o manuseio do produto. Injúrias mecânicas de impacto, vibração, compressão, abrasão e corte estão relacionadas a diversas alterações de composição química, física e sensorial em maçãs, pepinos, batatas e tomates. Hudson \& Orr (1977) observaram que existe relação direta entre a ocorrência de injúrias mecânicas de impacto e abrasão e perda de água em batatas.

Em tomates, a ocorrência de injúrias mecânicas de impacto nem sempre causa uma sintomatologia aparente, isto é, sinais externos e prontamente visíveis. 
Em alguns casos, a injúria mecânica manifesta-se apenas internamente, dando origem a desordem fisiológica de impacto. A ocorrência deste fenômeno foi inicialmente estudada por Halsey (1955), que observou amadurecimento alterado em frutos de tomate com esta desordem. A ocorrência da desordem fisiológica de impacto é cumulativa durante as diversas etapas de manuseio, e sua severidade depende da cultivar, estádio de maturação e número de impactos sofridos pelo fruto (McColloch, 1962, Sargent et al., 1989; Sargent et al., 1992).

Embora exista uma considerável quantidade de trabalhos abordando os efeitos de injúrias mecânicas sobre a fisiologia, metabolismo, aparência e qualidade de produtos hortícolas submetidos a injúrias mecânicas, há uma lacuna na literatura no que diz respeito à utilização de equipamentos eletrônicos na detecção da alteração de características químicas e físicas de frutos de tomate com desordem fisiológica de impacto. A detecção eletrônica de aromas tem sido amplamente utilizada nos últimos anos para a classificação de grãos (Borjesson et al., 1996), classificação de peixes armazenados (Di Natale et al., 1996), determinação do ponto de colheita em melões cantaloupe (Benady et al., 1995), determinação da qualidade de "blueberries" (Simon et al., 1996) e concentração de compostos voláteis em frutos de tomate maduros em relação ao ponto de colheita (Maul et al., 1998). O interesse por tecnologias não destrutivas tem aumentado a cada ano. Assim é que técnicas como medida de firmeza por aplanação (Calbo \& Nery, 1995), transmissão de impulsos acústicos na determinação da maturidade de melões (Sugyama et al., 1994) e fluorescência de pigmentos clorofílicos (DeEll, et al., 1997) têm se mostrado ferramentas efetivas para definir e predizer a qualidade de produtos frescos. Apesar do trabalho com estes tipos de ferramentas ter sido intenso nos últimos anos, a utilização de técnicas nãodestrutivas para a avaliação da ocorrência de danos mecânicos em produtos hortícolas ainda não foi avaliada.

O presente trabalho teve por objetivo avaliar a utilização de tecnologia nãodestrutiva denominada "nariz eletrônico" na discriminação de frutos de tomate com e sem desordem fisiológica causada por impacto.

\section{MATERIAL E MÉTODOS}

Material vegetal. Frutos de tomate (Lycopersicon esculentum Mill.), da cv. Solar Set, foram colhidos no primeiro semestre de 1997 em campos de produção comercial em Bradenton (Flórida, EUA), para o primeiro experimento, e na estação experimental da Universidade da Flórida (Gainesville, EUA), para um segundo experimento. Após a colheita, os frutos foram colocados em badejas de isopor (Niles Packaging, Michigan, EUA), similares às usadas para transportar ovos e levados para o laboratório de pós-colheita no mesmo dia. Após seleção para retirada de frutos danificados e classificação por tamanho (63 a $72 \mathrm{~mm})$ e massa (140 \pm 10 $\mathrm{g})$, os frutos foram tratados com $100 \mathrm{~mL}$. $\mathrm{L}^{-1}$ de etileno gasoso num sistema de fluxo contínuo (fluxo de $50 \mathrm{~mL} . \mathrm{s}^{-1}$ ) a $20^{\circ} \mathrm{C}$. Após atingirem o estádio de amadurecimento verde-rosado (menos de 10\% da superfície do fruto com coloração amarelo-tanino ou avermelhada), os frutos foram retirados do tratamento com etileno e submetidos a injuria mecânica de impacto.

Injúria mecânica de impacto e armazenamento. Os frutos foram submetidos à queda de uma altura de 0,40 m sobre uma superfície metálica, plana e rígida. Cada fruto sofreu dois impactos em pontos eqüidistantes de uma linha equatorial imaginária, tentando-se não atingir a parede locular que separa dois lóculos adjacentes. Após o impacto, os frutos foram armazenados a $20^{\circ} \mathrm{C}$ com $85-90 \%$ de umidade relativa até atingirem a maturidade comercial para os padrões de firmeza do mercado americano. O padrão foi determinado quando os tomates colocados sobre uma superfície côncava de borracha apresentaram uma deformação maior ou igual a $3 \mathrm{~mm}$ quando submetidos a uma força estática de 9,8 $\mathrm{N}$ aplicada por 5 segundos sobre a região equatorial do fruto. A ponta de prova utilizada possuía formato convexo e $11 \mathrm{~mm}$ de diâmetro. A firmeza foi medida com um medidor de firmeza do tipo Cornell (Hamson, 1952), adaptado por Gull et al. (1980).
Teste com o "nariz eletrônico". Ao atingirem o padrão anteriormente determinado, os frutos foram submetidos ao teste com o nariz eletrônico (modelo eNose 4000, Neonotronics Scientific Co., Flowery Branch, Georgia, EUA). O equipamento é, como o próprio nome sugere, uma simulação do sistema olfativo humano. Basicamente, ele consiste de uma série de 12 sensores químicos não específicos, cada um mostrando uma resposta distinta aos compostos voláteis que são liberados pelo produto objeto de estudo (Neonotronics Scientific Inc., 1996). A resistência elétrica dos sensores químicos muda à medida que a concentração de compostos voláteis no espaço amostral aumenta. As mudanças na resistência elétrica são traduzidas em números que são então analisados por um sistema de reconhecimento, utilizando-se como ferramenta, por exemplo, a análise de funções discriminantes.

A calibração do sistema, feita com polietileno glicol (PEG), durou $10 \mathrm{mi}$ nutos e foi feita em três etapas sendo monitorada por um microprocessador acoplado ao equipamento. Inicialmente, o frasco de amostragem foi aspergido com ar purificado (fluxo de $7 \mathrm{ml} . \mathrm{s}^{-1}$ ) por 2 minutos a fim de eliminar-se qualquer odor estranho à análise a ser feita. Procedeu-se então a aspersão de ar purificado por 4 minutos sobre os 12 sensores de polímeros condutores contidos na sonda de amostragem com a finalidade de eliminar-se qualquer composto que porventura estivesse impregnado nos sensores. Finalmente, os sensores foram rebaixados para o interior do frasco de amostragem e foram expostos por 4 minutos aos compostos voláteis liberados pelo PEG para calibrar o equipamento.

Após a calibração, os frutos injuriados e não-injuriados foram colocados, individualmente, no frasco de coleta do equipamento e as três etapas descritas anteriormente foram desenvolvidas, tendo-se dessa vez os frutos como elementos emanadores de compostos voláteis.

$\mathrm{O}$ delineamento empregado foi inteiramente casualizado com 2 tratamentos (frutos com desordem fisiológica causada por impacto e controle) e 8 repetições, sendo um fruto por repetição. 


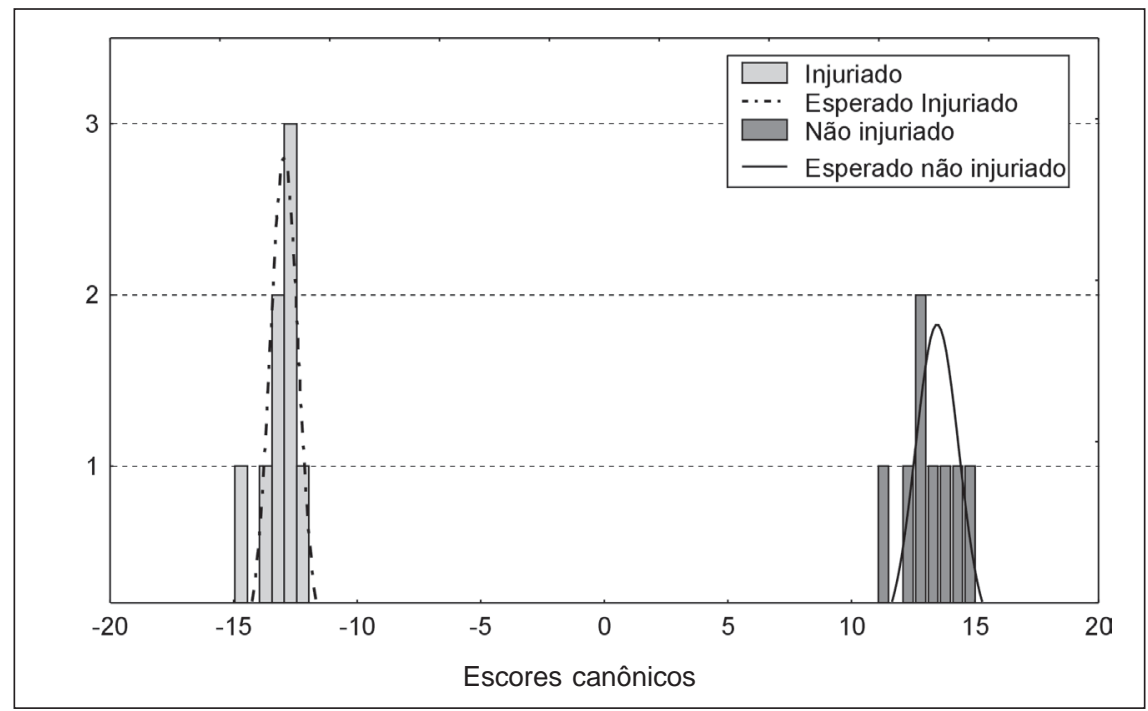

Figura 1: Frequência de escores canônicos para tomates 'Solar Set' com desordem fisiológica causada por impacto e controle. Gainesville, Flórida (EUA), Universidade da Flórida, 1997.

Os resultados apresentados são as médias dos dois experimentos conduzidos. Os resultados das alterações nas resistências elétricas dos 12 sensores empregados foram armazenados numa base de dados e posteriormente analisados. Utilizou-se a técnica de análise de funções discriminantes empregando-se o pacote de programas STATISTICA (Statsoft, 1994). Este procedimento estatístico de análise multivariada cria funções discriminantes lineares que maximizam as diferenças entre tratamentos e seus respectivos controles, otimizando a probabilidade de uma re-classificação tendo por base a concentração de compostos voláteis de uma amostra da qual não se conhecesse a história pregressa. Foi calculada a distância de Mahalanobis (distância entre os centróides de dois grupos distintos) a fim de comparar-se a extensão da diferença (grau de dissimilaridade) entre os tratamentos.

\section{RESULTADOS E DISCUSSÃO}

O "nariz eletrônico" mostrou-se uma tecnologia eficaz na detecção de frutos com desordem fisiológica de impacto. Ele forneceu informações suficientes para que o procedimento de análise discriminante multivariada encontrasse diferenças significativas $(\mathrm{P}<0,0041)$ entre os frutos injuriados e não injuriados. A distância de Mahalanobis $(28,19$ unidades) (Figura 1) entre os dois grupos de frutos foi um indicativo das dra- máticas diferenças encontradas pelos sensores ao amostrarem os compostos voláteis que foram produzidos pelos frutos. Segundo a análise discriminante multivariada, os frutos injuriados foram agrupados com escores canônicos variando entre -15 e -12 e os não injuriados entre 11 e 15 (Figura 1). A reclassificação dos frutos baseada na análise discriminante foi $100 \%$ acurada, isto é, a probabilidade de classificar-se um fruto qualquer como sendo injuriado ou não injuriado foi igual a 1,0.

Acredita-se que as alterações fisiológicas e metabólicas observadas nos frutos com desordem fisiológica de impacto possam estar relacionadas com as diferenças observadas. Os aumentos transientes da evolução de gás carbônico e etileno (Moretti et al., 1998), alterações na concentração de pigmentos carotenóides, considerados precursores de vários compostos voláteis em tomates (Buttery \& Ling, 1993), de compostos voláteis chave na determinação de aroma e sabor (Moretti et al., 1997), de ácidos orgânicos, vitamina $\mathrm{C}$ total e consistência (Moretti et al, 1998) podem estar relacionadas com os resultados detectados pelo equipamento.

A interpretação da distância de Mahalanobis foi útil para contrastar o grau de diferença encontrado entre os frutos que sofreram injúria mecânica de impacto e os frutos não injuriados. A distância obtida entre os grupos de frutos injuriados e não injuriados $(28,19$ unidades) foi quase três vezes maior do que a distância entre frutos intactos no estádio verde-rosado e vermelho $(10,72$ unidades) observada por Maul et al. (1997). Assim, foi possível colocar em perspectiva a importância do manejo pós-colheita na obtenção de frutos com sabor e aroma desejáveis.

Acredita-se que compostos voláteis como 2-isobutiltriazol, 1-penteno-3-ona, hexanal, cis-3-hexenal, entre outros, tenham papel importante na percepção de sabor de tomates (Kader et. al, 1977; Petró-Turza, 1987). Os resultados obtidos com esta tecnologia mostram a potencialidade de sua utilização na determinação não-destrutiva da qualidade de tomates frescos em indústrias e supermercados, com um custo relativamente menor e maior rapidez comparativamente às técnicas analíticas. $\mathrm{O}$ aroma e o sabor dos frutos submetidos à análise com o "nariz eletrônico" poderiam contar a história da vida pós-colheita do fruto quando nenhum sintoma visual, como no caso da desordem fisiológica causada por impacto, for perceptível. Desta forma, assegurar-se-ia que um maior número de frutos chegasse com melhor qualidade à mesa do consumidor.

\section{LITERATURA CITADA}

BENADY, M.; SIMON, J.E.; CHARLES, D.J.; MILES, G.E. Fruit ripeness determination by electronic sensing of aromatic volatiles. Transactions of the ASAE, v. 38, n. 1, p. 251257, 1995.

BORJESSON, T.; EKLOV, T.; JONSSON, A.; SUNDGREN, H.; SCHNURER, J. Electronic nose odor classification of grains. Cereal Chemistry, v. 73, n. 4, p. 457-61, 1996.

BUTTERY, R.G.; LING, L.C. Volatile compounds of tomato fruit and plant parts: relationship and biogenesis. American Chemistry Society, Symposium Series, Washington D.C., v. 525, p. 23-34, 1993

CALBO, A.G.; NERY, A.A. Medida de firmeza em hortaliças pela técnica de aplanação. Horticultura Brasileira, Brasília, v. 12, n. 1, p. 14-18, 1995.

DeELL, J.R.; PRANGE, R.K.; MURR, D.P. Chlorophyll fluorescence as na indicator of apple fruit firmness. In: International Conference on Nondestructive Techniques for Measuring Quality of Fresh Fruits and Vegetables, 1, 1997, Orlando, FL, Resumos...Orlando: ASAE, 1997, p. 1-7.

Di NATALE, C.; BRUNINK, J.A.J.; BUNGARO, F.; DAVIDE, F.; DAMICO, A.; PAOLESSE, R.; BOSCHI, T.; FACCIO, M.; FERRI, G. Recognition of fish storage time by metalloporphyrins-coated QMB sensor array. Measurement Science and Technology, v. 7, n. 8, p. 1103-1114, 1996. 
GULL, D.; CARTAGENA, D.; FRENCH, E.C. Análisis de calidad de tomato para lograr um mejor producto. IBTA, PRODES, UFLA, Cochabamba, Bolivia, 1980. 20 p.

HALSEY, L.H. Preliminary studies of bruising of 'turning' and 'pink' tomatoes caused by handling practices. Proceedings of the Florida State Horticultural Society, v. 68, p. 240243, 1955.

HAMSON, A.R. Measuring firmness of tomatoes in a breeding program. Proceedings of the American Society for Horticultural Science, v. 60, p. 425-433, 1952.

HUDSON, D.E.; ORR, P.H. Incidence of mechanical injuries to potatoes during certain storage-related handling operatios in the river valley production area. American Potato Journal, v. 54, p. 11-21, 1977.

KADER, A.A.; STEVENS, M.A.; ALBRIGHTHOLTON, M.; MORRIS L.L.; ALGAZI, M. Effect of fruit ripeness when picked on flavor and composition in fresh market tomatoes. Journal of the American Society for Horticultural Science, v. 102, n. 6, p. 724-731, 1977.

MAUL, F.; SARGENT, S.A.; BALABAN, M.O.; BALDWIN, E.A.; HUBER, D.J.; SIMS, C.A. Aroma volatile profiles from ripe tomatoes are influenced by physiological maturity at harvest: an application for electronic nose technology. Journal of the American Society for Horticultural Science, v. 123, n. 6, p. 1094 $-1101,1998$.
MAUL, F.; SARGENT, S.A.; HUBER, D.J.; BALABAN, M.O.; LUZURIAGA, D.A.; BALDWIN, E.A. Non-destructive quality screening of tomato fruit using "Electronic Nose" technology. Proceedings of the Florida State Horticultural Society, v. 110, p. 191-194, 1997.

McCOLLOCH, L.P. Bruising injury of tomatoes. Washington: USDA, 1962. 35 p. (USDA Marketing research report, 513).

MORETTI, C.L.; SARGENT, S.A.; HUBER, D.J.; CALBO, A.G..; PUSCHMANN, R. Chemical composition and physical properties of pericarp, locule and placental tissues of tomatoes with internal bruising. Journal of the American Society for Horticultural Science, v. 123, n. 4, p. 656-60, 1998.

MORETTI, C.L., SARGENT, S.A.; BALDWIN, E.; HUBER, D.J.; PUSCHMANN, R. Pericarp, locule and placental tissue volatile profiles are altered in tomato fruit with internal bruising. In: Congresso Brasileiro de Fisiologia Vegetal, 1, 1997, Belém, PA, Resumos.. Belém: SBFV, 1997, p. 216.

NEONOTRONICS SCIENTIFIC (Flowery Branch, GA). An introduction to electronic nose technology. Flowery Branch: 1996. 112 p.

PETRÓ-TURZA, M. Flavor of tomato and tomato products. Food Reviews International, v. 2, n. 3, p. 309-351, 1987
SARGENT, S.A; BRECHT, J.K.; ZOELLNER, J.J. Assessment of mechanical damage in tomato packing lines. Transactions of the $A S A E$, v. 35, n. 2, p. 235-245, 1989.

SARGENT. S.A.; BRECHT, J.K.; ZOELLNER, J.J. Sensitivity of tomatoes at mature green and breaker ripeness stages to internal bruising. Journal of the American Society for Horticultural Science, v. 117, n. 1, p. 119-23, 1992.

SIMON, J.E.; HERTZRONI, A.; BORDELON, B.; MILES, G.E.; CHARLES, D.J. Electronic sensing of aromatic volatiles for quality sorting of blueberries. Journal of Food Science, v. 61, n. 5, p. 967-969, 1996.

SUGIYAMA, J.; OTOBE, K.; HAYASHI, S.; USUI, S. Firmness measurement of muskmelons by acoustic transmission. Transactions of the ASAE, v. 37, n. 4, p. 12351241, 1994.

STATSOFT Inc. Statistica for Windows version 4.5. Tulsa, 1994. 890 p. (Tulsa Oklahoma). 